\title{
A simultaneous closed loop current calculation method for multiple distribution lines
}

\author{
Fang-Di Hou ${ }^{\dagger}$ \\ College of Electrical Engineering, Zhejiang University, \\ Hangzhou, 310027, China \\ ${ }^{\dagger}$ E-mail: houfangdi@zju.edu.cn \\ Bo Que, Lei Chen, Wei-Yan Zhen and Jun-Qiang Fu \\ Power Dispatch and Control Center, Hangzhou Electric Company, \\ Hangzhou, 310027, China
}

\begin{abstract}
In practical use, closed loop operation on distribution lines may generate large steady current and impact current. For multiple distribution lines, closed loop operations in turn with low load transfer efficiency are easy to lead black-out in large area. In order to increase efficiency without reducing reliability and security, this paper proposed a simultaneous closed loop current calculation method to support simultaneous closed loop operations by generating simultaneous admittance matrix. In this paper, a practical case is tested to prove the accuracy and superiority of the proposed simultaneous closed loop method for multiple distribution lines.
\end{abstract}

Keywords: Closed Loop Current; Simultaneous Closed Loop Operation; Simultaneous Admittance Matrix.

\section{Introduction}

The closed loop operation in distribution network is important for power dispatching when considering assurance of power supply reliability, facility maintenance and load transfer [1]. In practical use, closed loop operation may generate large steady current and impact current because of mismatching electrical components such as transformers or transmission lines and variance of load distribution. Coupled with closed loop current and load current, it may cause power exceeding equipment limit and misoperation of relay protection equipment, which severely affect the reliability of power supply and the safety of system [2]-[4].

In order to improve the feasibility of the closed loop operation, dispatchers have to calculate the closed loop current previously in simulation and then reduce the actual closed loop current by adjusting devices according to calculated results [5]. 
Currently, distribution power networks with $110 \mathrm{kv}$ and below in China adopt the mode of ring network design and open loop operation and transfer the load by closed loop operation with no power outrage [6]. Closed loop current calculation in distribution networks in China is mainly based on the off-line model, in which most closed loop operations on multiple distribution lines are one by one in a certain order to avoid overlarge current. However, closed loop operations in turn with low load transfer efficiency are easy to lead black-out in large area [7].

In order to increase efficiency without reducing reliability and security of closed loop operations on multiple distribution lines, this paper proposes a simultaneous closed loop current calculation method to simulate closed loop operations on multiple lines simultaneously. By using this proposed calculation method, closed loop current of simultaneous operations is lower than that with closed loop operations in turn. Thus, by using this method, dispatchers can adopt simultaneous closed loop operation according to the results of previous calculation.

The remainder of this paper is structured as follows: Section II shows the mathematic model of this proposed model. Section III introduces the system function and procedure of this method. In section IV, an example is discussed to verify the effectiveness of this method. The conclusion is drawn in Section V.

\section{Mathematical Models}

\subsection{Single distribution line closed loop model}

In theory, the calculation of closed loop current in the single distribution line mainly uses superposition principle. According to the superposition principle, the closed loop current in each branch is divided into two parts. One of them is initial current before closed loop operation, and the other is balanced current caused by voltage vector differential between closed loop switches.

Assume that the simplified equivalent closed loop circuit of regional power grid is shown in Figure 1. Before closed loop operation, it can be viewed as connecting a voltage source $\dot{U}_{i j}$ between the node $\mathrm{i}$ and $\mathrm{j}$ as shown in Figure 1(b). When it happens, the short circuit between the node $\mathrm{i}$ and $\mathrm{j}$ is equivalent to connect a reverse voltage source $-\dot{U}_{i j}$ to pull up voltage differential to zero as shown in Figure 1(a). According to superposition principle, initial current results from joint effect of the voltage source $\dot{U}_{i j}$ and sources in active network corresponding to the situation before closed loop operation. Balanced current is only generated by the reverse voltage source $-\dot{U}_{i j}$. In order to calculate closed loop current, we use substitution theorem to replace the reverse voltage source 
$-\dot{U}_{i j}$ by the equivalent current source $\dot{I}_{s}$ in Figure $1(\mathrm{~d})$, which is exactly the closed loop current.

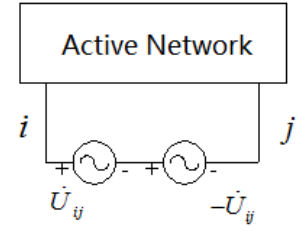

(a)

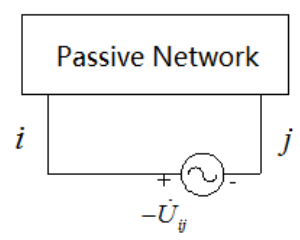

(c)

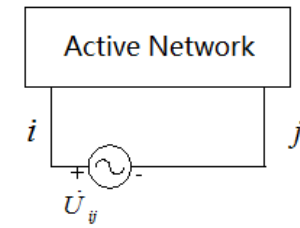

(b)

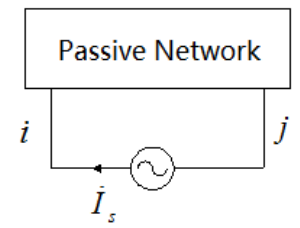

(d)

Fig. 1. Superposition principle on closed loop current calculation.

For the power network with $\mathrm{N}$ nodes, if network structure and element parameter are given, we can introduce the network equation as follows:

$$
Y \dot{U}=\dot{I},
$$

where $Y$ is $\mathrm{N} \times \mathrm{N}$ order nodal admittance matrix, $\dot{U}$ is $\mathrm{N} \times 1$ order nodal voltage column vector, $\dot{I}$ is $\mathrm{N} \times 1$ order nodal injection current column vector.

Changing (1) into incremental type results the following network equation:

$$
Y \Delta \dot{U}=\Delta \dot{I}
$$

From Figure 1(d), it can be seen that the equivalent current source injects current $I_{s}$ at node $\mathrm{i}$ and current $-I_{s}$ at node $\mathrm{j}$. Therefore, it follows that

$$
Y\left[\begin{array}{c}
\vdots \\
\Delta \dot{U}_{i} \\
\vdots \\
\Delta \dot{U}_{j} \\
\vdots
\end{array}\right]=\left[\begin{array}{c}
\vdots \\
1 \\
\vdots \\
-1 \\
\vdots
\end{array}\right] \dot{I}_{s} .
$$

Then (3) can be rewritten as 


$$
Y\left[\begin{array}{c}
\vdots \\
\Delta \dot{U}_{i} / \dot{I}_{s} \\
\vdots \\
\Delta \dot{U}_{j} / \dot{I}_{s} \\
\vdots
\end{array}\right]=\left[\begin{array}{c}
\vdots \\
1 \\
\vdots \\
-1 \\
\vdots
\end{array}\right] .
$$

With network structure and element parameter given, we can calculate $\Delta \dot{U}_{i} / \dot{I}_{s}, \Delta \dot{U}_{j} / \dot{I}_{s}$ by solving Eq. (4).

Voltage differential $\dot{U}_{i j}$ between node $\mathrm{i}$ and $\mathrm{j}$ can be given by means of topology analysis and state estimation before closed loop operation and it becomes zero after the operation. Therefore,

$$
\Delta \dot{U}_{i j}=\Delta \dot{U}_{i}-\Delta \dot{U}_{j}=-\dot{U}_{i j}
$$

According to (4) and (5), we have closed loop current vector:

$$
\dot{I}_{s}=-\frac{\dot{U}_{i j}}{\left(\Delta \dot{U}_{i} / \dot{I}_{s}-\Delta \dot{U}_{j} / \dot{I}_{s}\right)}
$$

\subsection{Multiple distribution lines closed loop model}

Suppose these switches are closed at the same time when simultaneous closed loop on multiple lines occurs. There are no apparent interaction effect for voltage differential $U_{i j}$ of these switches before closed loop operation. So simultaneous closed loop on multiple lines can be viewed as multiple closed loops on single distribution line. With only changing the network admittance matrix, we can calculate the current on each closed loop switch respectively by using the method on single distribution line repeatedly.

In multiple lines model, closed loop current on other lines would increase sharply when closed loop occurs on two nodes in the same substation. Therefore, this method eliminates the situation of two nodes closed loop in the same substation, which reflects on the change of network admittance matrix.

Compared with the model in single distribution line, the simultaneous admittance matrix of multiple lines $Y^{\prime}$ adds admittance values of multiple distribution lines on which closed loop operations would occur simultaneously. For the situation of two nodes closed up in the same substation, their admittance values would be set to zero in matrix $Y^{\prime}$.

Let the simultaneous admittance matrix be modeled as 


$$
Y^{\prime}=Y+\Delta Y,
$$

where $Y^{\prime}$ is $\mathrm{N} \times \mathrm{N}$ order simultaneous admittance matrix, $\Delta Y$ is $\mathrm{N} \times \mathrm{N}$ order variable admittance matrix and $\Delta Y$ can be written as

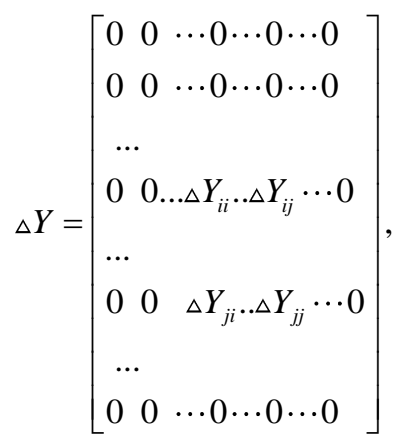

where $\Delta Y_{i i}$ is self-admittance variation of node $\mathrm{i}, \Delta Y_{i j}$ is off-diagonal elements variation of node $\mathrm{j}$ that is closed loop with node $\mathrm{i},{ }^{\Delta} Y_{j j}$ is selfadmittance variation of node $\mathrm{j}$. Therefore

$$
\Delta Y_{i i}=\sum_{j=1}^{n} y_{i j},
$$

$$
\begin{gathered}
\Delta Y_{i j}=\Delta Y_{j i}=-y_{i j}, \\
\Delta Y_{j j}=y_{j j},
\end{gathered}
$$

where $\mathrm{n}$ is the number of nodes that are closed loop with node $\mathrm{i}$. If node $\mathrm{i}$ and node $\mathrm{j}$ are in the same substation, $y_{i j}=0$

Substituting $Y^{\prime}$ into (4) results in the following equation

$$
Y^{\prime}\left[\begin{array}{c}
\vdots \\
\Delta \dot{U}_{i} / \dot{I}_{s} \\
\vdots \\
\Delta \dot{U}_{j} / \dot{I}_{s} \\
\vdots
\end{array}\right]=\left[\begin{array}{c}
\vdots \\
1 \\
\vdots \\
-1 \\
\vdots
\end{array}\right],
$$

By solving equation (12), we can calculate $\Delta \dot{U}_{i} / \dot{I}_{s}, \Delta \dot{U}_{j} / \dot{I}_{s}$ on each closed loop line. According to (6), current vector on each simultaneous closed loop line can be obtained in turn. 


\section{Solution Methodology}

\subsection{Topology analysis}

The size and position of load in power distribution network influence the distribution of power grid voltage, which creates voltage differential on closed loop switches. When closed loop operation happens, voltage differential produces huge loop current. So topology analysis in power distribution network is necessary in order to form the node-branch model before the calculation of closed loop current.

As the basis of solving analysis and calculation problem in power system, the main task of topology analysis is to process switch information in order to form new network structure and transform bus-switch physical model into nodebranch mathematical model.

In topology analysis, this method converts connected relations in power system physical model into graphs in graph theory by using matrix method, such as buses corresponding to nodes and switches to edges. In this way, adjacency matrix and incidence matrix exhibit related information of nodes with nodes and nodes with edges respectively.

\subsection{Voltage vector differential estimation}

In real power system, the calculation of closed loop current relates closely to voltage vector differential between closed loop switches. Besides the voltage amplitude, it needs to estimate the voltage phase angle differential at first.

In voltage phase angle differential estimation, it's difficult to require closed loop phase angle from SCADA system directly, because no measurements for phase angle are equipped on closed loop switches. This method adopts active power measurements to calculate closed loop voltage phase angle, which could improve the reliability and calculation speed of the algorithm. When estimating voltage phase angle, we ignore the change of the voltage amplitude, the P.U. value of which is taken as 1 .

For branches with active power measurements, it follows that:

$$
P_{i j}=B_{i j}\left(\theta_{i}-\theta_{j}\right)
$$

where $\mathrm{i}$ is the number of start node in this branch; $\mathrm{j}$ is the number of end node in this branch; $P_{i j}$ is the active power from start node $\mathrm{i}$ to end node $\mathrm{j} ; B_{i j}$ is the imaginary part of line I column $\mathrm{j}$ element in the node admittance matrix; $\theta_{\mathrm{i}}, \theta_{\mathrm{j}}$ are the voltage phase angle of node $\mathrm{i}$ and node $\mathrm{j}$. We note that the measurement equation of voltage phase angle differential estimation is the linear function of the phase angle as state variable. Therefore, this estimation problem 
can be viewed as weighted linear least squares estimation problem with high reliability and limited iteration.

In order to estimate voltage vector differential, we have to obtain voltage amplitude between closed loop switches after voltage phase angle differential estimation. Bus switches are generally equipped with voltage amplitude measurements so that voltage amplitude can be easily obtained. As for component switches, voltage amplitude measurements are equipped on the side of bus other than the side of component. We can estimate voltage amplitude on the side of component by using voltage estimator with node voltage amplitude measurements and imaginary part measurements of zero injection current.

\subsection{Modeling procedure}

The procedure of this simultaneous closed loop method for multiple distribution lines can be summarized as the following steps in Figure 2.

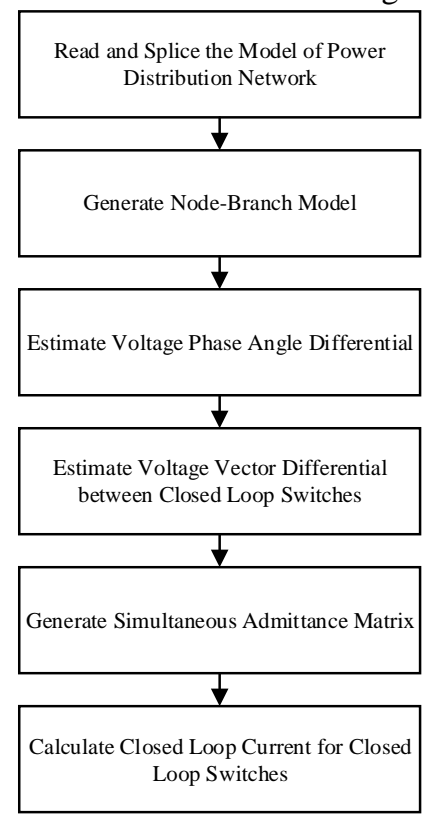

Fig. 2. Flow chart of the modeling process.

Step 1: Read and splice the model of power distribution network including the topology and measurement data of this model,

Step 2: Generate node-branch model according to the model of power distribution network,

Step 3: Estimate voltage phase angle differential using active power measurements in (13), 
Step 4: Estimate voltage vector differential between closed loop switches with estimated voltage phase angle differential,

Step 5: Generate simultaneous admittance matrix of this model in (7),

Step 6: Calculate closed loop current for closed loop switches using (12), (5) and (6).

\section{Illustrated Case}

In order to demonstrate the accuracy and superiority of the proposed simultaneous closed loop method for multiple distribution lines, a practical case is given here. The data of this case is based on the distribution network structure of province $\mathrm{H}$ in China. For privacy protection, we can only give rough information to simulate the practical case.

Figure 3 shows the distribution network structure of province $\mathrm{H}$ in this case. It can be seen that there are 2 pieces of $10 \mathrm{kv}$ bus bar in Substation AB. Bus I and Bus II are separately operated on Node A and Node B. As for Node A, there are 5 hand in hand feeder lines connecting to Bus I, including closed loop with Node B on Shuangliu 218 Connecting Line, closed loop with Node C on Beibu 216 Connecting Line, closed loop with Node D on Lingjia 319 Connecting Line, closed loop with Node E on Lilong 613 Connecting Line and closed loop with Node F on Huangshao 608 Connecting Line. In these 5 hand in hand feeder lines, only Shuangliu 218 Connecting Line connects two bus nodes in one substation.

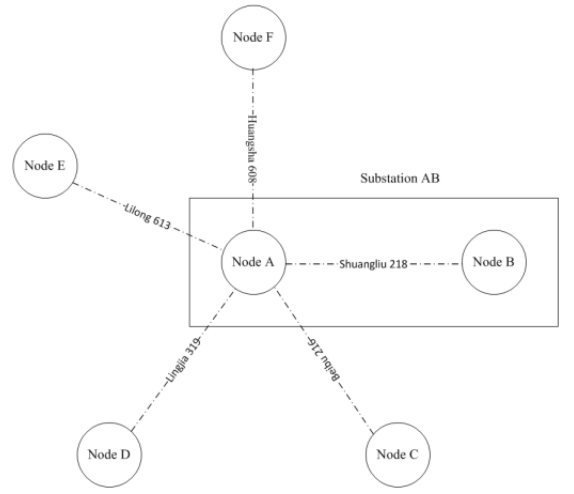

Fig. 3. Distribution network structure of province $h$ in this case.

Table 1 shows the closed loop current adopting closed loop operation on single distribution line in turn. The rank of closed loop on these distribution lines is Huangsha 608 Connecting Line, Beibu 216 Connecting Line, Lingjia 319 Connecting Line, Lilong 613 Connecting Line, Shuangliu 218 Connecting Line. The interval time of each closed loop operation is 2 seconds. 
Table 1. Closed loop current adopting closed loop operation on single distribution line in turn

\begin{tabular}{|c|c|c|c|c|}
\hline Rank & $\begin{array}{c}\text { Start } \\
\text { Time(s) }\end{array}$ & Switch & $\begin{array}{c}\text { Closed } \\
\text { Loop Line }\end{array}$ & Closed Loop Current(Amp) \\
\hline 1 & 0 & Switch AF & Huangsha 608 & 229.253001 \\
\hline 2 & 2 & Switch AC & Beibu 216 & 180.488255 \\
\hline 3 & 4 & Switch AD & Lingjia 319 & 413.152435 \\
\hline 4 & 6 & Switch AE & Lilong 613 & 314.337832 \\
\hline 5 & 8 & Switch AB & Shuangliu 218 & 19.533196 \\
\hline
\end{tabular}

In Table 1, it can be seen that the highest closed loop current on these 5 distribution lines is up to 413 Amp on Lingjia 319 Connecting Line. Reversely, Shuangliu 218 Connecting Line with two bus nodes in the same substation is the lowest closed loop current with only 19 Amp.

Table 2 shows the closed loop current adopting simultaneous closed loop operation on all 5 distribution lines.

Table 2. Closed loop current adopting simultaneous closed loop operation on all 5 distribution lines

\begin{tabular}{|c|c|c|c|}
\hline Start Time(s) & Switch & Closed Loop Line & Closed Loop Current(Amp) \\
\hline 0 & Switch AF & Huangsha 608 & 229.253001 \\
\hline 0 & Switch AC & Beibu 216 & 160.658809 \\
\hline 0 & Switch AD & Lingjia 319 & 360.333040 \\
\hline 0 & Switch AE & Lilong 613 & 272.021915 \\
\hline 0 & Switch AB & Shuangliu 218 & 496.082426 \\
\hline
\end{tabular}

Comparing Table 1 and Table 2, it can be seen that closed loop current on all the distribution lines with simultaneous closed loop operation is lower than that with operation in turn except Shuangliu 218 Connecting Line between Node $\mathrm{A}$ and Node $\mathrm{B}$ in the same substation. In theory, when adopting simultaneous closed loop operation, element $\mathrm{y}_{\mathrm{i}, \mathrm{j}}$ in the simultaneous admittance matrix is taken as 0 for nodes in the same substation so that current on these nodes may increase largely. Therefore, simultaneous closed loop operation should avoid closed loop on nodes in the same substation.

Table 3 shows the closed loop current adopting simultaneous closed loop operation on all distribution lines without nodes in the same substation. 
Table 3. Closed loop current adopting simultaneous closed loop operation on all distribution lines without nodes in the same substation

\begin{tabular}{|c|c|c|c|}
\hline Start Time(s) & Switch & Closed Loop Line & Closed Loop Current(Amp) \\
\hline 0 & Switch AD & Lingjia 319 & 316.257956 \\
\hline 0 & Switch AE & Lilong 613 & 204.675050 \\
\hline 0 & Switch AC & Beibu 216 & 103.668838 \\
\hline 0 & Switch AF & Huangsha 608 & 164.779634 \\
\hline
\end{tabular}

In Table 3 it can be seen that closed loop current on all the distribution lines without nodes in the same substation is lower than that in Table 2. Therefore, with avoiding closed loop on nodes in the same substation, this simultaneous closed loop method can effectively reduce the closed loop current.

\section{Conclusion}

This paper proposes a simultaneous Closed loop current calculation method for multiple distribution lines. Compared with closed loop operations in turn, simultaneous closed loop operations increase efficiency of load transfer without decrease system reliability and security. Case study has proved that closed loop current calculated in this proposed model of simultaneous operations is lower than that with alternate operations when avoiding closed loop on nodes in the same substation. The previous calculation of this proposed model also supports the simultaneous closed loop operations in practical use.

\section{References}

1. Tsai-Hsiang, Chen Wei-Tzer Huang, Jyh-CherngGu, Guan-Chih Pu, YenFeng Hsu, and Tzong-YihGuo, "Feasibility study of upgrading primary feeders from radial and open-loop to normally closed-loop arrangement," IEEE Trans. on Power Systems, vol. 19, pp. 1308-1316, Agu. 2004.

2. YE Qing-hua, TANG Guo-qing, WANG Lei, et al., "Development and application of the analysis system for closed-loop operation of distribution network," Automation of Electric Power Systems, vol. 26, pp. 66-69, 2002.

3. XIA Xiang, XIONG Jun, and HU Lie-xiang, "Analysis and control of loop power flow in regional power network," Power System Technology, vol. 28, pp. 76-80, 2004.

4. LI Su-su, "Analysis and control of the loop closing current based on power flow calculation in distribution network," M.S. thesis, Dept. Elect. \&Electron. Eng. North China Electric Power Univ., Beijing, China, 2006.

5. Zhelin Deng, Baozhu Liu and Munan Wang, "Closed-loop current adjustment strategy in distribution network based on sensitivity analysis," 
Power Engineering and Automation Conference (PEAM), 2011 IEEE, Wuhan, 2011, pp. 55-58.

6. YU Jian-hui, ZHOU Hao, LU Hua, "Research on hangzhou $10 \mathrm{kV}$ distribution network closing loop operation," Mechanical \& Electrical Engineering Magazine, vol. 10, 2007.

7. XING Rong-xia, YAO Ai-ming, "Analysis of Closed Loop Operation in Power Network,” Zhejiang Electric Power, vol. 01, 2007. 